EPJ Web of Conferences 56, 02004 (2013)

DOI: $10.1051 /$ epjconf/20135602004

(C) Owned by the authors, published by EDP Sciences, 2013

\title{
Modelling of the interaction between chemical and mechanical behaviour of ion exchange resins incorporated into a cement-based matrix
}

\author{
M. Neji ${ }^{1,2}$, B. Bary ${ }^{1}$, N. Burlion ${ }^{2}$ and P. Le Bescop ${ }^{1}$ \\ ${ }^{1}$ CEA, DEN, DPC, SECR, Laboratoire d'Etude du Comportement des Bétons et des Argiles, F- \\ 91191 Gif-sur-Yvette, France \\ 2 Polytech Lille - LML UMR 8107, Bd Paul Langevin, 59650, Villeneuve d'Ascq, France
}

\begin{abstract}
In this paper, we present a predictive model, based on experimental data, to determine the macroscopic mechanical behavior of a material made up of ion exchange resins solidified into a CEM III cement paste. Some observations have shown that in some cases, a significant macroscopic expansion of this composite material may be expected, due to internal pressures generated in the resin. To build the model, we made the choice to break down the problem in two scale's studies. The first deals with the mechanical behavior of the different heterogeneities of the composite, i.e. the resin and the cement paste. The second upscales the information from the heterogeneities to the Representative Elementary Volume (REV) of the composite. The heterogeneities effects are taken into account in the REV by applying a homogenization method derived from the Eshelby theory combined with an interaction coefficient drawn from the poroelasticity theory. At the first scale, from the second thermodynamic law, a formulation is developed to estimate the resin microscopic swelling. The model response is illustrated on a simple example showing the impact of the calculated internal pressure, on the macroscopic strain.
\end{abstract}

\section{Introduction}

Nowadays, several ways exist to clean up a fluid containing (deleterious) ionic species. One of them consists in using ions exchange resins. These kinds of resins are built to purge fluid from undesirable molecules thanks to ion exchange processes. In that way, a reusable fluid and a solid waste are obtained, which are easier to manage. However, mainly because of the non-respect of osmotic equilibrium between the external and internal fluid, an ion exchange resin tends to swell or shrink during the ion exchange processes [1-3]. Here, we focus on the mechanical behavior of ion exchange resins; in this case, the swelling cannot be neglected. Today, several approaches exist to describe the swelling phenomenon [4-7], but none of them has been used to characterize the impact of this swelling may have when the IER are embedded into another materials, in our case a cement paste matrix. In fact, most of them use thermodynamics equilibrium between the external solution and the resin to calculate the pressure range. In this paper we use, to calculate the swelling pressure of the IER, a mathematical formulation derived from the work of Soldatov[8] where the external solution would correspond to the interstitial solution of the cement paste. In fact Soldatov relies on the Gibbs

This is an Open Access article distributed under the terms of the Creative Commons Attribution License 2.0, which permits unrestricted use, distribution, and reproduction in any medium, provided the original work is properly cited. 
energy minimization at equilibrium state and the related nullity of its derivation. This condition leads to an expression which links osmotic pressure to the ionic activities $[1,2,8]$. From this method, a lot of study have been developed which differ from each other by the way the variables are calculated [9-11]. For instance, halfway between Soldatov works and the Flory-Huggins theory, Maurer et al. [7] developed a method which, via again the minimization of the Gibbs energy, estimates the evolution of the volume fraction of the ion exchange resin. However, this formulation has the drawback to necessitate an important knowledge about the polymer constitution and fabrication, that's why this method has been left behind.

In the nuclear context, the solidification of polluted resins into a cement-based matrix is a standard solution for waste storage $[12,13]$. The knowledge of the mechanical behavior of this kind of composite is then of great importance. The IER are characterized by an aptitude to change their volume depending on the type of ion present in the surrounding solution. The cement paste is a porous material with an ionic interstitial solution which is able to react with the IER. Obviously, the worst case might be an expansion of the resin into the cement matrix in such a way that it may potentially cause material degradation. In very specific conditions, this scenario has been validated by experimental observations[12,13]. With this in mind, our purpose is then to assess the resin swelling risks and to ensure that the generated internal pressures never exceed the material strength. To achieve this goal, we propose in this work to develop a predictive model, based on experimental data, which can determine the mechanical behavior of a material made up of ion exchange resins embedded into a CEM III cement paste. To estimate the microscopic swelling of the IER, a formulation based on existing approaches has been developed, which takes into account the cement matrix surrounding the resins. Next, the microscopic pressure effects have been upscaled to the macroscopic level via an analogy with the poroelasticity theory and the introduction of an interaction coefficient $[14,15]$. Analytical homogenization techniques have been applied for this purpose to estimate the mechanical homogenized properties and the interaction coefficient [16-19]. In that way, from the knowledge of the microscopic pressure we are able to determine the macroscopic mechanical response of the material.

\section{The composite structure}

This section reports briefly the necessary backgrounds for this study regarding ion exchange resin (IER). The resin employed for this study is an Amberlite IR120H from ROHM\&HAAS, a cationic IER [20]. This means that, during the ion exchange process, only cations can move from one environment to another. Besides, the Amberlite IR120H is a hydrogel, that is, its structure might be divided in two main parts [7]: a solid phase and an internal solution (Figure 1). The solid phase itself might be sub-divided in two sub-parts: a network of hydrophobic polymer chains called "the skeleton" and the functional groups, counter-ions where the cations could be attached $[1,21]$. The internal solution consists of (i) ionic species fixed to functional groups via an electromagnetic link and, (ii) a certain amount of water which contributes to the osmotic equilibrium. Moreover, two geometrical forms of IER are susceptible to be contained into the cement matrix $[1,13]$. IER are typically made in the form of spheres with size ranging from approximately 500 to $800 \mu \mathrm{m}$, as illustrated in Figure 2, but for storage or special applications, it could be interesting to crush them. The model developed needs then to accommodate both types of shape. 


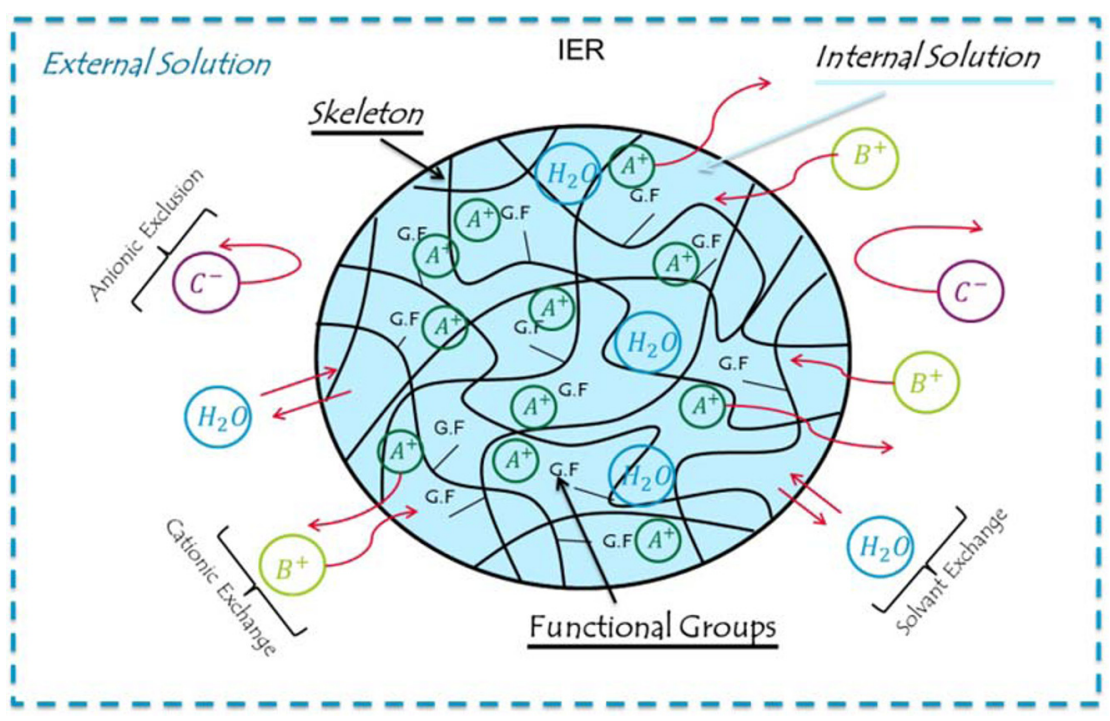

Fig. 1. Schematic illustration of a ion exchange resin with the external solution

\section{Microscopic swelling}

This section deals with the mathematical formulation which will allow estimating the evolutions of the resin swelling. The Figure 2 gives a schematic view of the IER. This view postulates that the internal solution of the IER (symbol ") behaves like a fluid phase encapsulated in an elastic structure where the role of the structure is similar to that of a semipermeable membrane in an osmotic equilibrium. The whole resin is embedded into a surrounding solution (symbol') where we consider the pressure as a constant. As explained in the introduction, several ways have been reported in the literature to study ion exchange processes. Most of them, as the one we chose, start from the figure 2 by writing the thermodynamic equilibrium based on the second principle of thermodynamics $[1,5,7]$.

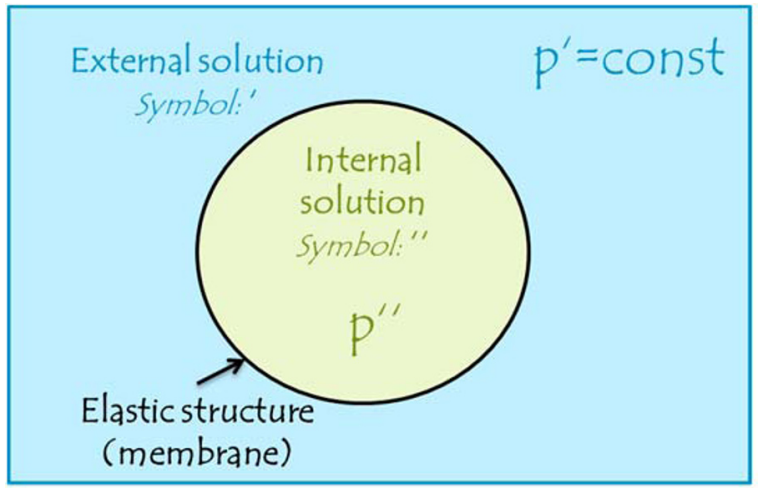

Fig. 2. Simplified schematic illustration of a ion exchange resin with the external solution 


\subsection{Thermodynamic equilibrium}

The second principle of thermodynamics may be expressed as:

$$
T d S-p d V \geq d U
$$

where $\boldsymbol{T}$ is the absolute temperature, $\boldsymbol{p}$ the pressure, $\boldsymbol{S}$ the entropy, $\boldsymbol{U}$ the internal energy and $\boldsymbol{V}$ the volume of the system.

In our situation, the system may be simplified according to the notation of figure 2 (i.e. the symbols " and ' designate the internal and external solution, respectively) and after various manipulations of the equation (1) we obtain, at the equilibrium state, the following equation [7]:

$$
d\left\{G^{\prime}\left(T, p^{\prime}, n^{\prime}\right)+G^{\prime \prime}\left(T, p^{\prime \prime}, n^{\prime \prime}\right)+A^{m}\left(T, V^{\prime \prime}\right)\right\}=0
$$

where $n$ is the total number of mole of ion, $G=U+p V-T . S$ is the Gibbs energy and $A^{m}=U-$ TS the Helmotz energy.

Equation (2) can be rewritten in terms of chemical potential as follow $[1,2,7,8]$ :

$$
\sum\left\{\mu_{i}^{\prime}\left(T, p^{\prime}, n^{\prime}\right)-\mu_{i}^{\prime \prime}\left(T, p^{\prime \prime}, n^{\prime \prime}\right)+\vartheta_{i}^{\prime \prime} \pi\right\} d n_{i}=0
$$

where $\mu_{i}=\mu_{i}{ }^{r e f}+\boldsymbol{R} \boldsymbol{T} \ln \left(\boldsymbol{a}_{\boldsymbol{i}}\right)$ is the chemical potential of species ' $\mathrm{i}$ ', $\mu_{\boldsymbol{i}}{ }^{\boldsymbol{r e f}}$ its reference chemical potential, $\boldsymbol{R}$ the gas constant, $\boldsymbol{a}_{\boldsymbol{i}}$ the chemical activity of the species 'i', $\boldsymbol{\vartheta}_{\boldsymbol{i}}$ its molar volume and $\boldsymbol{\pi}=\left(\boldsymbol{p}^{\prime \prime}-\boldsymbol{p}^{\prime}\right)$ is the osmotic pressure.

\subsection{Model and prediction}

The thermodynamic equilibrium has been set up, and now from the equation (3) we are able to express the osmotic pressure $\pi$ in the case of multicomponent systems. For an easier understanding, we consider in the following and without loss of generality a binary exchange between ions ' $A$ ' originally in the internal solution and ' $\mathrm{B}$ ' in the external solution.

\subsubsection{Equilibrium equation}

From equation (3), by considering the activity $a_{i}$ as the product of the activity coefficient $\gamma_{i}$ and the equivalent molar ratio $x_{i}$, we easily get in the case of the considered binary system[1]:

$$
\pi=R T \ln \left(\frac{K_{A B}}{\vartheta_{A}-\vartheta_{B}}\right)
$$

where the thermodynamic equilibrium constant $K_{A B}$ is expressed by:

$$
K_{A B}=\frac{x_{A}^{\prime} \frac{1}{Z_{A}} x_{B}^{\prime \prime \frac{1}{Z_{B}}}}{x_{A}^{\prime \prime \frac{1}{Z_{A}}} x_{B}^{\prime} \frac{1}{Z_{B}}}\left(\frac{\gamma_{B}^{\prime \prime}}{\gamma_{B}^{\prime}}\right)^{\frac{1}{Z_{B}}}\left(\frac{\gamma_{A}^{\prime}}{\gamma_{A}^{\prime \prime}}\right)^{\frac{1}{Z_{A}}}
$$

The coefficients introduced in the thermodynamic equilibrium constant expression (5) are detailed in the following subsections. 


\subsubsection{Activity coefficient in IER phase $\gamma_{i}^{\prime \prime}$}

Widely used in ion exchange process studies, the Wilson model is based on Gibbs energy which differs from the ideality $[9,22-26]$. The activity coefficient in the resin is expressed by the following relation in the case of the binary exchange:

$$
\gamma_{i}^{\prime \prime}=\exp ^{1-\ln \left[x_{i}^{\prime \prime}+\left(1-x_{i}^{\prime \prime}\right) \Lambda_{A B}\right]}-\left(\frac{x_{i}^{\prime \prime}}{x_{i}^{\prime \prime}+\left(1-x_{i}^{\prime \prime}\right) \Lambda_{A B}}\right)-\left(\frac{\left(1-x_{i}^{\prime \prime}\right) \Lambda_{B A}}{x_{i}^{\prime \prime} \Lambda_{B A}+\left(1-x_{i}^{\prime \prime}\right)}\right)
$$

where $\boldsymbol{\Lambda}_{\boldsymbol{A} B}$ and $\boldsymbol{\Lambda}_{\boldsymbol{B} \boldsymbol{A}}$ are the Wilson coefficients which are specific to a kind of IER and to a type of ionic exchange. They are determined via an experimental campaign.

\subsubsection{Activity coefficient in external solution $\gamma_{i}^{\prime}$}

The choice of an efficient method to calculate the activity coefficients in the external solution depends mostly on its ionic strength. In the context of the CEM III cement paste as considered in this study, the ionic strength does not exceed about $1 \mathrm{~mol} / \mathrm{L}$ [27]. In these conditions the B-dot model is known to be a good compromise between simplicity and accuracy. It is an extension of the DebyeHückel formulation for solutions with ionic strength around the unity [4,23]. The activity coefficient takes the form:

$$
\log \gamma_{i}^{\prime}=\left(\frac{-A_{B d o t} z_{i}^{2} \sqrt{I}}{1+B_{B d o t} r_{i} \sqrt{I}}\right)+\dot{B}_{B d o t} I
$$

where $A_{B d o t}, B_{B d o t}, \dot{B}_{B d o t}$ are the B-'dot' coefficients determined via an experimental campaign, $z_{i}$ the ionic charge of the species ' $\mathrm{i}$ ', $r_{i}$ its ionic radius and $I=\frac{1}{2} \cdot \sum z_{i}{ }^{2} \cdot C_{i}$ is the ionic strength, sum of the product obtained by multiplying the square of each ionic charge by its respective concentration $C_{i}$.

\subsubsection{Equivalent molar ratio $x_{i}^{\prime \prime}, x_{i}^{\prime}$}

Part of ions from the external solution will be exchanged with those which belong to the resin. This phenomenon might be attributed, in one hand, to the resin capacity and, in other hand, to the ionic couple which would be exchanged.

The existing link between equivalent molar ratio of ions in the external solution and in internal solution is determined via an 'ion exchange isotherm' (Figure 3). The ratio of ions which enter in the resin during the ion exchange process versus what had been initially in the external solution can be determined from the isotherm plot.

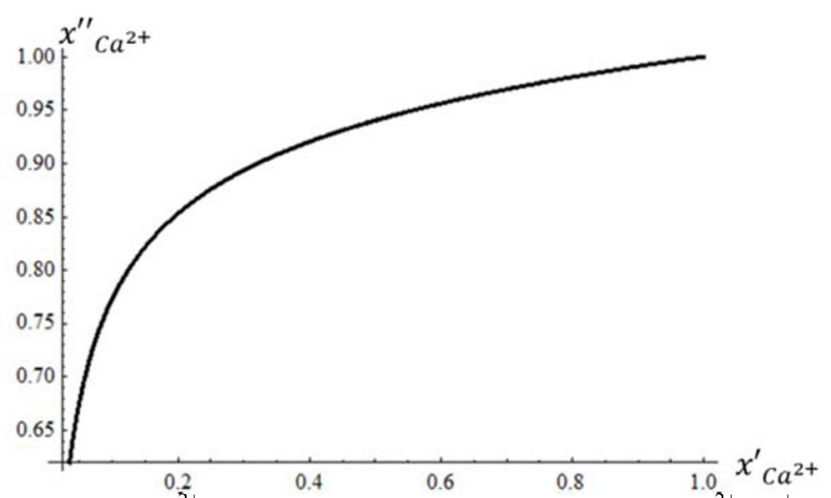

Fig. 3. $\mathrm{Ca}^{2+}$ ion exchange isotherm for the couple $\mathrm{Ca}^{2+} / \mathrm{Na}^{+}$ with a resin capacity of $1.810^{-3} \mathrm{eq} / \mathrm{g}$ 
In addition, 'the ion exchange isotherm' gives an idea about the resin affinity for any ionic species in the external solution. Indeed, for the same ionic form of the resin, bigger is the slope of the curve, more important the selectivity would be for the ion in comparison with another.

\section{Macroscopic modelling}

\subsection{Micro-macro upscaling}

From the formulation of the microscopic pressures established in the previous section, we develop a model for upscaling their effects at the macroscopic level. The composite is composed of swelling spherical IER embedded into a cement paste matrix; and we propose to apply a homogenization method to upscale the microscopic swelling effects of the IER at the level of the REV (Figure 4).

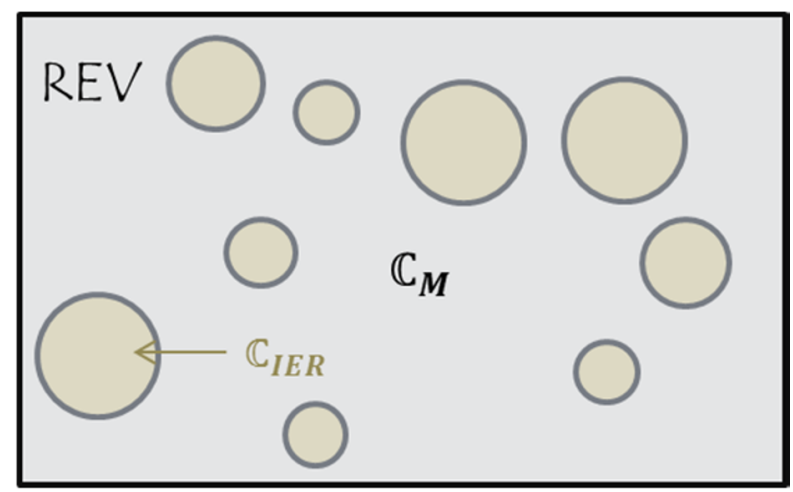

Fig. 4. REV of the materials composed of spherical IER embedded into a cement paste matrix.

To achieve that goal, a solution might be an analogy with the poroelasticity, as Lemarchand et al. [15] or Bary[14] did before in another context. In fact by introducing an interaction coefficient similar to the Biot coefficient, for an isotropic behavior the macroscopic stress tensor may be expressed as a function of the microscopic pressure and macroscopic strain in the form:

$$
\Sigma=\mathbb{K}_{h}: \varepsilon-\pi \cdot b . I
$$

where $\mathbb{K}_{h}$ is the homogenized fourth order stiffness tensor, $\boldsymbol{\Sigma}$ and $\boldsymbol{\varepsilon}$ are respectively the macroscopic stress and strain tensor and b is the interaction coefficient. $I$ corresponds to the second order identity tensor.

Analytical homogenization techniques are further applied to estimate the mechanical properties and the interaction coefficient from the characteristics and physical properties of the cement paste and resin phases. In view of the shape of the heterogeneities, i.e. spherical or ellipsoid-like, we make the choice to apply analytical homogenization schemes based on the Eshelby theory [16]. To be more specific, to take into account different kinds of shapes with an isotropic distribution, we select the double-inclusion type model called Interaction Direct Derivative (IDD)[19]. The specificity of this homogenization method is to model the spatial distribution by a spheroidal cell, called doubleinclusion, surrounding the inclusion[17-19]. In the next sections we briefly present the developments leading to the estimations of both $\mathbb{K}_{h}$ and $b$. 


\subsection{Homogenized stiffness tensor}

The chosen homogenization method derives from the Eshelby theory which characterizes the effects of the inclusion shape via the Eshelby tensor $\mathbb{S}_{\text {esh }}$. An important restriction of single inclusion homogenization methods is that we are generally not able to choose separately a kind of inclusion shape and a spatial distribution. As explained previously, the IDD is a double inclusion type model; this means that two Eshelby tensors are used, one for the inclusion shape and the other for describing the spatial distribution $[25,26]$, both selected independently. The first step is to calculate the average dilute strain localization tensor of IER inclusion as:

$$
\mathbb{T}_{I E R}=\left(\mathbb{I}-\mathbb{S}_{\mathrm{esh}}\left(\mathbb{I}-\mathbb{C}_{I E R} \mathbb{C}_{0}^{-1}\right)\right)^{-1}
$$

where II is the fourth order identity tensor, $\mathbb{C}_{I E R}$ and $\mathbb{C}_{0}$ are the stiffness tensor of the IER and the cement paste, respectively. Next we do the same operation for the spatial distribution with the slight difference that the Eshelby tensor $\mathbb{S}_{\text {Desh }}$ describes the spatial distribution. Thus we obtain the average dilute strain localization tensor of IER spatial distribution $\mathbb{T}_{D I E R}$.

From these two tensors, it is possible to estimate the homogenized stiffness tensor as [18]:

$$
\text { With }\left\{\begin{array}{c}
\mathbb{K}_{h}=3 \cdot k^{\mathrm{IDD}} \mathbb{J}_{h}+2 \cdot \mu^{\mathrm{IDD}} \mathbb{L}_{d} \\
k^{\mathrm{IDD}}=k_{0}\left(1+\frac{\phi_{I E R} \cdot T_{I E R}^{h}\left(1-\frac{k_{I E R}}{k_{0}}\right)}{1-\phi_{I E R}\left(\Xi_{I E R}{ }^{h}-T_{I E R}{ }^{h} \frac{k_{I E R}}{k_{0}}\right.}\right)^{-1} \\
\mu^{\mathrm{IDD}}=\mu_{0}\left(1+\frac{\phi_{I E R} T_{I E R}^{d}\left(1-\frac{\mu_{I E R}}{\mu_{0}}\right)}{1-\phi_{I E R}\left(\Xi_{I E R}{ }^{d}-T_{I E R}{ }^{d} \frac{\mu_{I E R}}{\mu_{0}}\right)}\right)^{-1}
\end{array}\right.
$$

where $\mathbb{I}_{\boldsymbol{h}}$ and $\mathbb{L}_{\boldsymbol{d}}$ are the hydrostatic and deviatoric projection operator, $\boldsymbol{T}_{\boldsymbol{i}}{ }^{\boldsymbol{h}}$ and $\boldsymbol{T}_{\boldsymbol{i}}{ }^{\boldsymbol{d}}$ are the hydrostatic and deviatoric projection of $\mathbb{T}_{I E R} \cdot \boldsymbol{\Xi}_{i}{ }^{\boldsymbol{h}}$ and $\boldsymbol{\Xi}_{\boldsymbol{i}}{ }^{\boldsymbol{d}}$ correspond to the hydrostatic and deviatoric projection of the product $\left(\mathbb{T}_{\text {IER }}\left(\mathbb{T}_{\text {DIER }}\right)^{-\mathbf{1}}\right)$ and $\boldsymbol{\phi}_{\text {IER }}$ is the volume ratio of IER [18]. Concerning the mechanical properties, $\boldsymbol{k}_{I E R}, \mu_{I E R}$ are the bulk and the shear modulus of the resin, respectively, while $\boldsymbol{k}_{\mathbf{0}}, \mu_{\mathbf{0}}$ are the ones associated to the cement paste.

\subsection{Coefficient of interaction}

One advantage of analytical upscaling procedures as used here is that they can also be applied for estimating rigorously, from the same microstructure representation as for $\mathbb{K}_{h}$, the interaction coefficient appearing in Equation.(8). Based on previous works by Dvorak \& Benveniste [28] and extended latter by among others Bary and Lemarchand et al $[14,15]$, it is shown, for an isotropic behavior, that the interaction coefficient may take the form:

$$
b=\phi_{\mathrm{IER}} \mathbb{A}_{\mathrm{IER}}^{T}: \mathbb{I}: \mathbb{I}
$$

where $\mathbb{A}_{\text {IER }}^{T}$ is the strain localization tensor of the IER which is calculated here with the IDD homogenization scheme. 


\subsection{Illustration}

In this study, we have developed a mathematical formulation to determine the macroscopic mechanical response of a material made up of ion exchange resins embedded into a CEM III cement paste. The Figure 5 shows an illustration of the impact of internal pressure on the macroscopic bulk stress $\boldsymbol{\Sigma}_{\text {macro }}=-\pi . b . \boldsymbol{I}$. This figure has been determined for three different volume fractions of IER, in the case of $\mathrm{Na}^{+} / \mathrm{K}^{+}$exchange and based on nano-indentation campaign for mechanical properties and on experimental data from[26] for chemical quantities. As expected, we observe that the amplitude of $\Sigma_{\text {macro }}$ strongly depends on the microstructure of the material, and in particular on the IER volume fraction.

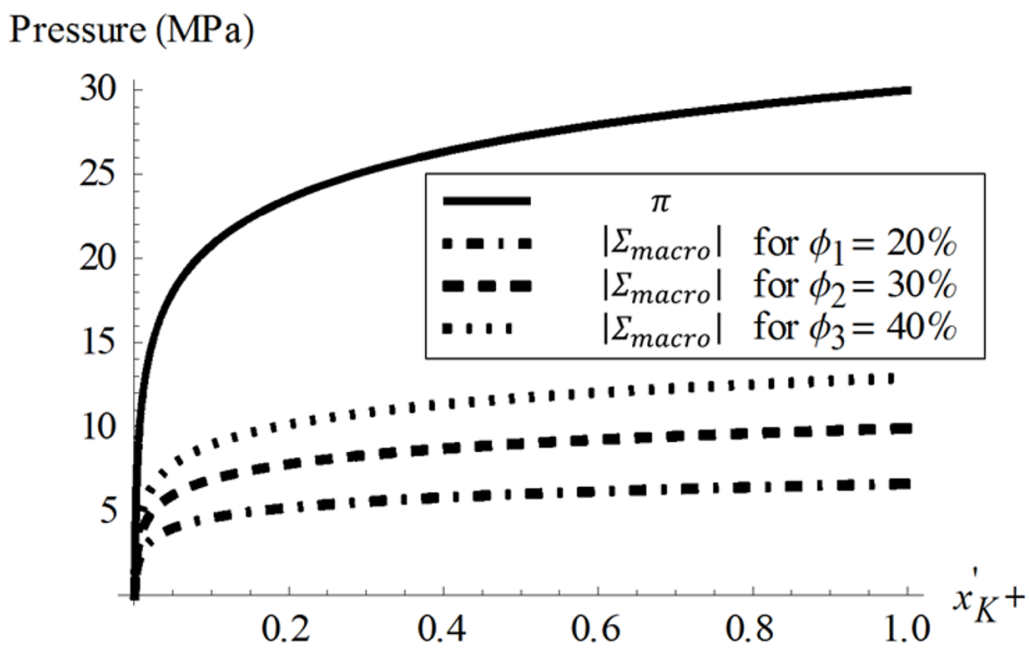

Fig. 5. Illustration of the evolution of macroscopic stress $\mid$ Emacro $\mid$ and osmotic pressure $\pi$ versus equivalent molar ratio $x_{K}^{\prime}$

\section{Conclusions}

The model development is still in progress. The next step of the study will consist in analyzing the effects of the ionic form of the resin, its capacity and the composition of the cement paste interstitial solution on the pressure generation, and in comparing it to experimental data. Furthermore, the homogenized stiffness tensor (section 4.2) and the coefficient of interaction (section 4.3) estimated by the analytical homogenization method will be confronted to numerical results obtained by $3 \mathrm{D}$ finite element simulations so as to justify and validate the chosen scheme. Finally, when the model will be considered as fully operational, a specific experimental program will allow to validate it by measuring the overall stresses and strains on a specimen subjected to interstitial solutions containing various ionic species concentrations.

\section{Acknowledgements}

The authors are deeply grateful to AREVA for its financial support and L. Stefan from AREVA for her support of the project. 


\section{References}

[1] A. Zagorodni, Ion exchange materials, first edition, Properties and Applications, 2007.

[2] F. Helfferich, Ion exchange, McGraw-Hill, 1962.

[3] J.M. Prausnitz, R.N. Lichtenthaler, Gomes de Azeved, Molecular thermodynamics of fluidphase equilibria, Third Edition, 1999.

[4] F. Gressier, Etude de la rétention des radionucléides dans les résines échangeuses d'ions des circuits d'une centrale nucléaire à eau sous pression, Manuscrit de thèse, Ecole des Mines de paris, 2008.

[5] H. Vink, Thermodynamics of ion exchange equilibria in polyelectrolyte systems, J. Chem. Soc. 81 (1985) 1677-1684.

[6] H.P. Gregor, Gibbs-Donnan equilibria in ion exchange resin systems, Journal of the American Chemical Society. 73 (1951) 642-650.

[7] G. Maurer, J.M. Prausnitz, Thermodynamics of phase equilibrium for systems containing gels, Fluid phase equilibria. 115 (1996) 113-133.

[8] V. Soldatov, Application of basic concepts of chemical thermodynamics to ion exchange equilibria, Reactive and Functional Polymers. 27 (1995) 95-106.

[9] A. de Lucas, J.L. Valverde, M.C. Romero, J. Gómez, J.F. Rodríguez, The ion exchange equilibria of $\mathrm{Na}+/ \mathrm{K}+$ in nonaqueous and mixed solvents on a strong acid cation exchanger, Chemical engineering science. 57 (2002) 1943-1954.

[10] A.. Gantman, A mathematical model for mixed-diffusion dynamics of ion-exchange sorption, Russian journal of physical chemistry. 69 (1995) 1652-1655.

[11] E. Hogfeldt, Ten years experience of a simple three-parameter model to fit ion exchange data, Reactive polymers. 11 (1989) 199-219.

[12] M. Matsuda, T. Nishi, K. Chino, M. Kikuchi, Solidification of spent ion exchange resin using new cementitious material,(I), Journal of Nuclear Science and Technology. 29 (1992) 883889.

[13] D. Chartier, Cimentation de résines échangeuses d'ions : Etude Bibliographique DTCD/SPDE/2008/16, CEA, 2008.

[14] B. Bary, Simplified coupled chemo-mechanical modeling of cement pastes behavior subjected to combined leaching and external sulfate attack, Int. J. Numer. Anal. Meth. Geomech. 32 (2008) 1791-1816.

[15] E. Lemarchand, L. Dormieux, F.-J. Ulm, Micromechanics investigation of expansive reactions in chemoelastic concrete, Philosophical Transactions of the Royal Society A: Mathematical, Physical and Engineering Sciences. 363 (2005) 2581-2602.

[16] E. Stora, Multi-scale modeling and simulations of the chemo-mechanical behavior of degraded cement-based materials, Manuscrit de thèse, Université Paris-Est, 2007.

[17] E. Stora, Q.C. He, B. Bary, Influence of inclusion shapes on the effective linear elastic properties of hardened cement pastes, Cement and concrete research. 36 (2006) 1330-1344.

[18] B. Bary, Estimation of poromechanical and thermal conductivity properties of unsaturated isotropically microcracked cement pastes, International Journal for Numerical and Analytical Methods in Geomechanics. 35 (2011) 1560-1586.

[19] Q.S. Zheng, D.X. Du, An explicit and universally applicable estimate for the effective properties of multiphase composites which accounts for inclusion distribution, Journal of the Mechanics and Physics of Solids. 49 (2001) 2765-2788.

[20] Rohm\&Haas, Data Sheet AMBERLITE IR120H, 2008.

[21] F. Dardel, Echange d'ions : Principe de base, Techniques de l'ingénieur, 2000.

[22] G.M. Wilson, Vapor-liquid equilibrium. XI. A new expression for the excess free energy of mixing, Journal of the American Chemical Society. 86 (1964) 127-130.

[23] B.S. Vo, D.C. Shallcross, Modeling Solution Phase Behavior in Multicomponent Ion Exchange Equilibria Involving $\mathrm{H}+, \mathrm{Na}+, \mathrm{K}+, \mathrm{Mg} 2+$, and $\mathrm{Ca} 2+$ Ions, Journal of Chemical \& Engineering Data. 50 (2005) 1995-2002. 
[24] J.L. Valverde, A. de Lucas, M. González, J.F. Rodríguez, Equilibrium data for the exchange of $\mathrm{Cu} 2+, \mathrm{Cd} 2+$, and $\mathrm{Zn} 2+$ ions for $\mathrm{H}+$ on the cationic exchanger Amberlite IR-120, Journal of Chemical \& Engineering Data. 47 (2002) 613-617.

[25] J.L. Valverde, A. de Lucas, M. González, J.F. Rodríguez, Ion-exchange equilibria of Cu2+, $\mathrm{Cd} 2+, \mathrm{Zn} 2+$, and $\mathrm{Na}+$ ions on the cationic exchanger Amberlite IR-120, Journal of Chemical \& Engineering Data. 46 (2001) 1404-1409.

[26] A. De Lucas, J. Zarca, P. Ca, Ion-exchange equilibrium of $\mathrm{Ca} 2+, \mathrm{Mg} 2+, \mathrm{K}+, \mathrm{Na}+$, and $\mathrm{H}+$ ions on Amberlite IR-120: experimental determination and theoretical prediction of the ternary and quaternary equilibrium data, Separation science and technology. 27 (1992) 823-841.

[27] E. Lafond, Etude de l'évolution chimique des résines échangeuses d'ions en milieu cimentaire - Influence sur l'hydratation du liant, LP2C Marcoule, 2012.

[28] G.. Dvorak, Y. Benveniste, On Transformation Strains and Uniform Fields in Multiphase Elastic Media, 437 (1992) 291-310. 